\title{
ARTÍCULOS
}

\section{TIC y educación en tiempos difíciles}

\section{IC'T and Education in Difficult Times}

\author{
Guzmán de Castro, Belkys Juliana; Santiago Castro; Acosta Cruz, William \\ Duvan
}

(D) Belkys Juliana Guzmán de Castro
Universidad Pedagógica Experimental Libertador,
Venezuela
belkys.juliana.guzman@gmail.com
(D) Santiago Castro
Universidad Pedagógica Experimental Libertador,
Venezuela
castrosantiago2015@gmail.com
(D) William Duvan Acosta Cruz
Ude Cataluña,Colombia
wacostaupelipc@gmail.com

Delectus

Instituto Nacional de Investigación y Capacitación Continua, Perú ISSN-e: 2663-1148

Periodicidad: Semestral

núm. 2, 2021

publicaciones.iniccperu@gmail.com

Recepción: 16 Febrero 2021

Aprobación: 18 Junio 2021

Publicación: 01 Julio 2021

Esta licencia permite a otros entremezclar, ajustar y construir a partir de su obra con fines no comerciales, y aunque en sus nuevas creaciones deban reconocerle su autoría y no puedan ser utilizadas de manera comercial, no tienen que estar bajo una licencia con los mismos términos.

\section{c) (i) (3)}

Esta obra está bajo una Licencia Creative Commons Atribución- NoComercial 4.0 Internacional.
Resumen: Este artículo tiene por objeto el análisis de la situación de la educación en Latinoamérica en momentos de pandemia del coronavirus. Se presentan reflexiones y consideraciones que se han tomado o debido tomar en cuenta para hacer los cambios que la contingencia actual ha generado por desconocimiento del contexto y las realidades de cada país, Se hará una descripción sucinta de la interrelación de los diferentes elementos del sistema, los cambios realizados y las consecuencias que se han observado. La información fue generada a partir de entrevistas informales realizadas a docentes y revisión crítica de artículos. Los resultados no pueden ser más disimiles. En el país se tiene dos ejemplos básicos pasando de resultados favorables en algunas universidades como la metropolitana hasta otros ejemplos no tan positivos como las universidades públicas, por los problemas de conectividad y formación de la comunidad educativa.

Palabras clave: Pandemia; Tecnologías de Información y Comunicación; Educación

Abstract: This article aims to analyze the situation of education in Latin America at times of the coronavirus pandemic. Reflections and considerations that have been taken or should be taken into account are presented to make the changes that the current contingency has generated due to ignorance of the context and the realities of each country. A succinct description will be made of the interrelation of the different elements of the system, the changes made and the consequences that have been observed. The information was generated from informal interviews with teachers and critical review of articles. The results could not be more dissimilar. In the country there are two basic examples, going from favorable results in some universities such as the metropolitan one to other examples that are not as positive as public universities, due to the problems of connectivity and training of the educational community.

Keywords: Pandemic; Information and Communication Technologies; Education.

\section{INTRODUCCIÓN}

El confinamiento, el poco intercambio que hay entre vecinos y compañeros de estudio o de trabajo de manera presencial ha obligado a expertos y a otros no tan expertos a volcarse hacia la cercanía mediada por dispositivos. Las llamadas, video llamadas y mensajería de texto o voz son la forma expedita que se ha encontrado para comunicarse y acercarse a familiares y amigos. Hoy día la obsesión de estar comunicados y conectados mediante diversos dispositivos y el tener todo o casi todo nuestro día a día en Instagram, Twitter o YouTube, compartiendo o almacenando en la nube propicia y permite adaptarse al entorno cambiante en el cual las necesidades de comunicación deben ser satisfechas.

Los países a raíz de la pandemia según la UNESCO (2020) han tenido tres campos de acción principales: el despliegue de modalidades de aprendizaje a distancia, mediante la utilización de una diversidad de formatos y plataformas (con o sin uso de 
tecnología digital); el apoyo y la movilización del personal y las comunidades educativas, y la atención a la salud y el bienestar integral.

En definitiva, el 2020 ha sometido a la población global a la modificación inminente de sus hábitos, rutinas de trabajo, de consumo y socialización. En tal sentido, la pandemia ha provocado innumerables medidas de precaución desde el uso de tapabocas, suspensión de actividades laborales, actividades escolares y comerciales, en fin; ha generado un colapso en poco tiempo que afectó física, mental y emocionalmente al mundo entero. Sin embargo, toda esta crisis catapultó a las Tecnologías de la Información y Comunicación (TIC) y su uso masivo como jamás se había pensado (Ojeda \& Palacios, 2021 p. 98).

Las TIC han impactado de manera importante a la sociedad, la cual realiza una transición en los aspectos sociales, económicos, políticos, culturales, educativo, entre otros, que a su vez repercuten en su estilo de vida y elevan su calidad de vida, pero también ha hecho evidente la brecha social y económica de una sociedad que necesita emplear todas las herramientas disponibles para satisfacer sus motivaciones e intereses de manera consciente y responsable (Reynosa Navarro et al., 2021). Así, es común observar, algunos docentes y estudiantes que se comunican todos los días a través de las redes instaurando la educación virtual de manera progresiva sin que el trauma de la obligatoriedad impacte de manera negativa los procesos de enseñanza y aprendizaje, es decir docentes estudiantes y padres sin la formación necesaria para enfrentar este reto.

Guzmán (2020); Amitabh, (2020) señalan que llueven las ofertas vía online, y varían desde links abiertos o no para videos y libros gratuitos, acceso a contenidos, cursos, tutorías, clases virtuales, entre otros que permiten paliar dificultades. Además, los docentes desempeñan diferentes roles entre ellos comunicar de forma efectiva, es por ello que deben contar con una capacitación en el uso de las diferentes herramientas digitales. Interactuando e intercambiando materiales como archivos de audios y video o grabar las sesiones, usando Google Meet, Zoom video o app y durante el tiempo que la conectividad de internet lo permita realizar intercambios de conocimientos y saberes.

Por otro lado, la UNESCO ha identificado grandes brechas en los resultados educativos, que se relacionan con una desigual distribución de los docentes, en general, y de los docentes mejor calificados, en particular, en desmedro de países y regiones con menores ingresos y de las zonas rurales, que suelen concentrar además a la población indígena y migrante (UNESCO, 2016a; Messina y García, 2020).

Para Villen (2020) la COVID-19 puso a prueba los desarrollos TIC cuando la educación mundial dependió de las herramientas y aplicaciones tecnológicas para el desarrollo dela educación virtual y al parecer lo tecnológico dio una respuesta aceptable, no así los docentes, ya que un porcentaje significativo independiente de la edad, demostraron que la preparación y formación sobre la competencia digital les llevó a experimentar emociones principalmente de sorpresa y una necesidad de formación en competencias digitales con las que puedan incrementar el uso de recursos en los procesos de formación a distancia. Estos docentes no estaban preparados pues la labor educativa desde el hogar trae muchos distractores y obstáculos y además multiplica el trabajo de los maestros, dejándoles poco tiempo para desarrollar nuevas destrezas digitales y nuevos dominios en las aplicaciones disponibles (García, 2020).

Aunado a lo anterior se tiene que los servicios de los diferentes países aumentan la desigualdad entre los ciudadanos, llegan de manera desigual en todas sus regiones y los estratos más bajos. Guzmán (2020) señala que al voltear la mirada a través de un cristal impregnado en ciencia y tecnología nos fijamos que en todos los tiempos se ha encontrado la manera de usar la tecnología a su servicio y satisfacer sus necesidades y ha usado lo descubierto por la ciencia para lograrlo, pero desafortunadamente no llega a todos los ciudadanos de la misma manera. Pero en estos momentos los estudiantes, profesores, quienes se han visto en la necesidad u obligación de utilizar las TIC para desarrollar los procesos académicos se encuentran que no basta con saber usar las TIC si no se sabe enseñar con ellas en el contexto donde los estudiantes deben aprender (Ferrada-Bustamante et al., 2021).

Existen grandes brechas tecnológicas en el acceso y uso de las TIC, con altos niveles de desigualdad entre segmentos socioeconómicos, grupos etarios, personas educadas y analfabetas, sin embargo, los docentes consideran que es un problema utilizar TIC en el contexto educativo, debido a que el confinamiento y la no presencialidad generan una realimentación más lenta entre estudiantes y profesores, en comparación a la que se produce en la presencialidad.

En la actualidad los investigadores, planificadores e innovadores educativos no se han detenido en trabajar en la alfabetización tecnológica y cuando muchos en los usos en los diferentes elementos del sistema de enseñanza y aprendizaje y la apropiación en TIC por parte de los ciudadanos. Los sistemas educativos de Iberoamérica tienen como común identificador la aspiración de "construir al ciudadano que requiere el país y que en muchos casos responde al perfil delineado en las constituciones nacionales" y el "desarrollo integral de la personalidad", y desde sus bases legales visualizan a un 
ciudadano con valores socioculturales, respetuoso de la paz, respeto a la vida, que sepa confrontar los problemas, encuentren soluciones para él, la comunidad y su país, un líder comunitario que conoce y participa en su ámbito promueve en los miembros de su entorno las vías para tener una vida mejor y más acceso al mercado laboral. Estos países han decidido en muchos casos dejando de lado la realidad y el contexto de cada una de sus regiones, integrar al día a día y al proceso educativo las TIC en forma obligatoria. La comunicación propicia la construcción colectiva y efectiva del conocimiento, para la producción de productos de calidad, propicia la intra e intersubjetividad haciendo consiente lo que conoces y aprendes, y favorece la interacción aprendiz-docente, entre pares y estudiantes con los medios para obtener un aprendizaje de efectivo y eficiente pasa de ser presencial a otras formas de presencia asumiendo que el mensaje llegará de igual manera (Guzmán, 2013); Guzmán \&Castro, 2020).

\section{METODOLOGÍA}

El estudio se refiere a una investigación de tipo documental basado en la revisión de fuentes bibliográficas relacionadas con el estado del arte de lo que diversos autores han aportado en relación con la educación en la pandemia con la finalidad de revisar los rasgos y posibilidades de estas herramientas para reconstruir el estado actual del problema educativo, este estudio se apoyó en entrevistas a docentes de diferentes países.

En su desarrollo se consideró la revisión de investigaciones de diferentes autores, entre las que figuran Marinoni et al. (2020); Puleo (2020); Ordorika,(2020); Picón, González de Caballero, \& Paredes Sánchez (2020); IESALC UNESCO (2020), para contrastar las percepciones individuales acerca del tópico y así identificar la existencia o no de puntos de coincidencia y de discenso entre sus enfoques.

Los criterios utilizados para la selección de documentos fueron específicamente que son originados de investigación y sus resultados se relacionan con las TIC y el problema educativo en esta época difícil de pandemia, se analizó y discriminó la información contenida en canales y/o artículos de revistas arbitradas relacionados con educación.

\section{RESULTADOS Y DISCUSIÓN}

La bibliografía seleccionada fue registrada en una matriz donde se plasmó el arqueo de las fuentes (artículos publicados enrevistas arbitradas) además del análisis y resumen de la información relevante y cuyos aportes son resumidos a continuación.

Se debe comenzar por señalar que las TIC, su uso y apropiación al proceso educativo, plantea cambios importantes en los fines de aquel y en el rol que desempeñan sus actores principales: estudiantes y docentes en el salón de clases, en la comunidad y por ende en un país o nación. Las naciones y el Estado tienen el deber y la responsabilidad de ofrecer una educación de calidad con pertinencia social, cultural y económica, en concordancia con las exigencias de la sociedad.

Pero en Venezuela este tiempo de desinformación, con poca o casi inexistente inversión en educación, infraestructuras ineficientes y con poca o ninguna seguridad, además con internet dentro y fuera de las escuelas es escaso 0 ausente, incluso sin teléfonos en muchas casas, surge la pregunta ¿la ausencia de equipos y condiciones de conectividad para operacionalizar las TIC en el currículo escolar. Es igual a nivel mundial, somos nosotros, o es otra cosa lo que preocupa? Es que, en estos ámbitos socioculturales, políticos y ambientales, la educación se sobresale, y a veces es una más del problema 0 de las consecuencias. Cuando pensamos en el mundo como sistema obtenemos que son muy pocos los países que han invertido en educación esta inversión es menor que para otros elementos del macrosistema.

La situación de los países de Latinoamérica es muy parecida no por tener un tronco común sino por el actuar de mucha de su gente, poca o ninguna libertad de expresión y comunicación, estallidos sociales, problemas con los ciudadanos y la guinda del pastel que son los problemas de salud que se han agravado con la pandemia.

En los diferentes países la pandemia, golpea en el rostro a los que conocían y a los que no querían conocer y daban la espalda a su realidad educativa. Esta añade un elemento más a la compleja y crítica situación de la educación que no solamente ocultan, sino que maquillaban para informes nacionales e internacionales. Prácticamente en todo el mundo se sabe que en América latina a los problemas no resueltos y en muchos casos ni siquiera enfrentados como la calidad, inequidad y la pérdida progresiva del presupuesto público para la educación, han mermado la formación del ciudadano con las competencias que se requiere. Para nadie es un secreto las elevadas tasas de abandono y el porcentaje de estudiantes que no completan la carrera. 
Para agravar la situación Piñero (2021) señala que en la lucha contra la COVID-19 en el contexto educativo venezolano les correspondió a los docentes nacionales transitar por esta situación, acompañada de muchas adversidades. Estos profesionales de la docencia, unos con formación en TIC y otros con capacidades reducidas o nulas en este mundo, les ha correspondido el reto de enseñar vía online. Si bien las TIC habían llegado para quedarse en las instituciones educativas, las características socioeconómicas de América Latina y especialmente de Venezuela muestran un contexto desigual, que es más perceptible en tiempos de pandemia (p.297).Esto refleja que se están viviendo tiempos difíciles, pero no es momento de detenernos a describir, ya lo observarán a través de toda la situación. Tiempo de cuarentena nacional y los estudiantes de escuelas y liceos siguen realizando sus actividades académicas, ahora de manera virtual. Esta medida sorprendió a las instituciones educativas públicas y privadas de Venezuela y otros países, que debieron definir una estrategia de contingencia de emergencia para acatar el decreto nacional, sin perder su cronograma escolar.

Ni ellos, ni muchos de nosotros reconocemos que al descuidar uno de los subsistemas, la entropía y la falta de equilibrio, hace tambalear hasta el país más desarrollado. En estos momentos la problemática de salud que impacta a la sociedad se agrava por la falta de prevención y planificación de lo que se haría para enfrentar el tener al estudiantado de todos los niveles y modalidades en sus casas, tratando de seguir su preparación para ser un profesional que participa en la sociedad de manera efectiva.

Ahora bien, en tiempos de estallidos sociales o pandemias, los estados y naciones han tomado la decisión de suspender las clases presenciales para toda la educación en sus diversos niveles y modalidades y en muchos casos se ha asumido la virtualidad y pasamos con niños y adolescentes en primaria y bachillerato de presencialidad a la distancia sin formación, ni motivación, nos imaginamos entonces un grupo de niños que aprenden de sus pares y que interactúan de diferentes maneras, aprendiendo unos de otros y de los docentes, y cuando algo no entienden levantan sus manos y preguntan en vivo y en directo, y estos ahora sin ninguna formación sino la que han aprendido solos o de sus padres deben interactuar no solo con los materiales sino que deben aprender solos y resolver sus problemas de aprendizaje con poca o ninguna interacción con el docente y sus pares.

El cambio a la enseñanza a distancia en todas las etapas tomó a la comunidad educativa con el pie izquierdo. 0 te adaptabas a las nuevas condiciones de enseñanza a distancia o perecías, en un contexto personal y social. Se dio por hecho que todos los contenidos y competencias podían impartirse online, que los padres y madres y acudientes podrían sustituir a los docentes y el hogar a la escuela, sin tomar en cuenta todas las emociones involucradas (Rogero-García, 2020);(Rujas\&Feíto,2021).

Se deben señalar dos ejemplos uno de la India y otro de Alemania, donde se presentan dos jóvenes que tienen acceso a los recursos tecnológicos, se sienten frustrados y molestos porque extrañan el contacto y la interacción directa con el docente, sus compañeros y su escuela.Es significativo entender que se cambian las condiciones del juego, los procesos de enseñanza y aprendizaje, aunque tienen el mismo propósito ya no se planifica ni administra de la misma manera, ahora es más complejo porque envuelve cambios en las prácticas, debe haber autodisciplina y compromiso con la didáctica y el aprendizaje. Aquí la voluntad juega un papel fundamental, pero se requiere la concientización docente, estudiantes, padres y representantes, es decir de la comunidad en general de la importancia de seguir con la formación, que hay una inversión de tiempo, dinero y esfuerzos por parte de todos.

Se requiere que docentes y estudiantes se familiaricen con las plataformas, las herramientas, recursos y posibilidades que ofrece, asimismo los recursos que tenemos para poder desarrollar el proceso formativo; y hacer un aprendizaje más dinámico de acuerdo con la audiencia. La virtualización supone un desafío para el quehacer docente, las instituciones, más aún cuando en muchos países se privilegia la presencialidad, sistema con el cual docentes y estudiantes se hallan habituados, pero aun cuando el modelo de enseñanza y aprendizaje utilizado es tradicional a través de clases magistrales, lo que genera sentimientos de angustia, desconfianza e incertidumbre lo que en muchos casos paraliza y no invita a la acción.

La educación es la clave para el desarrollo de cualquier nación y en Venezuela está consagrada como un derecho en la Declaración Mundial sobre Educación Superior (UNESCO, 1996, 1998), que se pretende impartir en esta época en muchos países y que tendrá carácter virtual cuando desarrollen el proceso de formación, actualización o capacitación exclusivamente a través de entornos virtuales de aprendizaje. La modalidad del sistema propone diálogos de saberes que permitan la inclusión y la formación del ciudadano fundamentada en todas las corrientes del pensamiento mediante la planificación, desarrollo y evaluación de programas de formación inicial y permanente, apoyada en la aplicación de las TIC al servicio del aprendizaje independiente y flexible del estudiante (CPEAD, 2011).

Para su ejecución es necesario: 
1. Conocer las experiencias de Institución en relación con:

a. Formación de docentes y estudiantes en relación con las TIC, TAC y TEP y especialmente los entornos virtuales de aprendizaje.

b. Uso de las aplicaciones: redes sociales, blogs, e-books, podcast, videos, historietas, sitios web, contenidos en formatos cd-rom, guías y libros impresos, radio y tv.

c. Uso y familiarización de los actores del sistema de aulas virtuales y plataformas.

2. Describir la interacción de docentes y estudiantes con los medios, recursos, estrategias de aprendizaje y de evaluación utilizados.

3. Identificar las fuentes de financiamiento para el uso, mantenimiento y alojamiento de los entornos virtuales.

4. Plataformas infotecnológica presentes en la escuela y en las casas de los participantes

Para desarrollar este trabajo se partió de la premisa que los sistemas de enseñanza y aprendizaje independientemente de la modalidad tienen seis subsistemas que se interrelacionan entre sí. Estos son: elementos directrices, propósitos, metas que deben estar claros y en función de las necesidades del país y el planeta, por ello nos dedicaremos a los otros subsistemas como son docente, estudiante, evaluación, estrategias, los medios y otros recursos.Aunque la poca o ninguna preparación de algunos de los actores.

Es importante tomar en cuenta que no todos los contenidos pueden verse a distancia, especialmente la práctica docente, la comisión de educación a distancia UPEL determinó que no eran temas para ser desarrollados a distancia pues de los estudiantes deben demostrar las competencias desarrolladas durante toda la carrera. Otro ejemplo son las habilidades y destrezas que se desarrollan en laboratorios de ciencias que son procedimentales y que a distancia solamente veremos cómo se medían contenidos conceptuales.

\section{Escenario}

La UNESCO señala que solamente con la pandemia se afecta la educación de más 1.500 millones de estudiantes a nivel mundial. Y si a eso se lea agregan algunos estallidos sociales, el número se pierde de vista. Según este organismo mundial, la pandemia ha conducido a tomar una serie de decisiones, en muchos casos, sin tomar en cuenta el contexto y la realidad del país. Los perfiles universitarios actuales deben satisfacer las necesidades de la sociedad e incluso proyectarlos.

Según datos de la UNESCO, en Europa, hoy por hoy, la única excepción a la regla del cese de actividades presenciales es Suecia. En Estados Unidos, las medidas están en manos de las autoridades estatales, pero la inmensa mayoría de campus, en particular los de grandes universidades públicas y privadas, habían cerrado semanas antes de la intervención del gobierno. Para el 31 de marzo de 2020, 185 países han cerrado escuelas y universidades de todo su territorio, migrando de una modalidad presencial hacia metodologías de enseñanza y aprendizaje virtuales.

En América Latina, Argentina, Chile, Colombia, Cuba, Perú, El Salvador. Uruguay, Venezuela Brasil y México; con la cuarentena total se suspendieron las clases presenciales en las IES a partir del 12 de marzo; incluso se ha pensado que, hasta el 4 de mayo, hoy, en pleno 2021, aún se estudia si se vuelve a clases presenciales o no.

Cada caso muestra una serie de variantes que incluyen desde educación virtual hasta asistida, hasta el uso de medios elaborados por los docentes (guías con el contenido y las tareas que los maestros entregan a los padres, estos las llevan a sus casas y luego de desarrolladas las reenvían a la escuela para su corrección. Este último lo están utilizando en algunas escuelas de Colombia que no han capacitado a los docentes para impartir clases virtuales y ni la escuela ni los estudiantes cuentan con acceso a internet de calidad, en sus casas solamente cuentan con radio y televisión, algún celular con plan de datos, entonces ejecutan otras maneras. Lo lamentable es que independientemente de la forma que se esté utilizando tiene a docentes, padres y representantes desesperados por la cantidad de tareas de ciencias, matemáticas y otros que envían a los niños complicando la situación que ya se tiene.

En América se habilitaron las plataformas digitales como las de Paraguay y Argentina, que se apoyan en diferentes recursos tecnológicos como al WhatsApp, videoconferencias, Classroom, mientras que en Chile: a)se ha desarrollado instructivos para los académicos, b) se han capacitado a docentes y estudiantes sobre cómo usar la plataforma tecnológica, programas y redes sociales, c) se ha crearon un Aula Virtual, en la que el profesor puede entregar material que enriquezca su clase, interactuar con los estudiantes y, eventualmente, impartir mediante videoconferencia. ¿Ahora todos los países se han dedicado a eso?

En Aprender Digital, una plataforma del Ministerio de Educación colombiano, hay más de 80.000 recursos educativos digitales, organizados por grados, que incluyen desde videos hasta aplicaciones y juegos. El país también ha comenzado a 
transmitir programas educativos en la radio y la televisión públicas, combinando una estrategia innovadora con otra más tradicional.

El informe de la UNESCO señala en relación con la conectividad de internet en los hogares son muy dispares en América Latina, con extremos en Chile y en Bolivia, siendo el primero el más alto y el segundo el más bajo. Mientras que las líneas móviles las estadísticas muestran que son más positivas que en el hogar lo que invitaría a desarrollar una enseñanza usando la telefonía celular. Desafortunadamente Venezuela es el segundo país más bajo solamente superamos a Cuba. Es decir, ni en nuestras casas ni por telefonía se cuenta con oportunidad objetiva para aprovechar las soluciones tecnológicas.

Cecodap (2020) refiere en su informe que el Observatorio Venezolano de Servicios Públicos en septiembre 2020, afirmó que $34 \%$ de los hogares tiene acceso a internet y en regiones del país como oriente (59.1), los andes (58.8), centro occidente $(57,1)$ y Zulia (54.2) hay una valoración de esta estrategia como deficitaria que supera la media global, esta es peor en los abuelos que en los padres o la observada en la región capital $(38,9)$. Este resultado se relaciona a las suspensiones del servicio eléctrico prolongadas y a las fallas del servicio de internet, especialmente en los estados del interior del país. El mismo informe señala que la firma Consultores 21 para marzo 2020, indica que el acceso a internet se reduce a 38\% de los hogares y que solamente $56 \%$ de las personas cuentan con teléfonos inteligentes. Aunado a esto se encuentra la falta de lineamientos claros y uniformes por parte del Ministerio para la Educación que permita desarrollar procesos de educación a distancia bajo criterios de calidad y especialmente adaptados en contexto que impone la pandemia y el peligro de destruir el futuro de los niños y jóvenes por la vulneración de su derecho a la educación (Vargas, 2021).

Toda institución que desee implementar la educación presencial, mixta 0 a distancia basada en las TIC debe gestionar políticas para la negociar la infraestructura necesaria, computadoras en red, servidores y una buena conexión a Internet con un ancho de banda adecuado a la demanda del proceso, sin restricciones impuestas a la comunidad que poco se basan en las necesidades administrativas y académicas de la comunidad.

La mayoría de las instituciones educativas de cualquier nivel en Latinoamérica no cuentan con plataforma infotecnológica, por diferentes razones bajo o ningún presupuesto para adquirir equipos, los que tienen muchos están desactualizados y en otros casos los laboratorios y equipos han sido despojados por el hampa.

Por otra parte, sus docentes tienen poca o ninguna experticia en el diseño de aulas virtuales basadas en las necesidades de la audiencia, medios en diferentes formatos adaptados a los contextos y las características de la audiencia o poco amigables, motivantes, no ofrecen un abanico de opciones para que el participante interactúe y construya su propio conocimiento, es decir no están preparadas para este cambio. Un ejemplo de ello son las instituciones de educación superior, en Paraguay que señalan que solo 10 de 46 universidades, tienen una plataforma online para sus estudiantes y cuentan con personal entrenado para su manejo. Esto también abarca a aquellas que ofrecen carreras de grado y postgrado bajo la modalidad de educación virtual.

Algunas instituciones han implementado el sistema de ingreso a las plataformas de información sincrónica o asincrónica, pero pocos son los docentes que tienen las competencias, para administrarlos propiciando que las iniciativas desde las instituciones de educación superior sean inválidas, porque deben considerar la actualización y capacitación de los profesores que no puede ni debe ser pasada por alto.

Para diseñar y ejecutar los procesos de enseñanza y aprendizaje se deben tomar en cuenta las características de la audiencia, los elementos directrices, las estrategias, medios y recursos más adecuados para mediar el aprendizaje, es por ello que para desarrollar las aulas virtuales y los medios instruccionales deben tener competencias para hacerlo, es decir deben ser docentes que toman en cuenta, las teorías de enseñanza de aprendizaje y también lo cognitivo y afectivo.

Como "no hay conexión a internet tenemos que ir a la escuela buscar las tareas entregarla a sus padres quien dos días después las traen y deben llevarse nuevas, lo que ha recargado de trabajo y angustia al padre", eso en el mejor de los casos pues la informante 3 (venezolana estrato muy bajo) señala que "los niños de la comunidad donde vive hace mucho tiempo que no saben lo que es entrar a un aula o tener algo relacionado con su educación".

La velocidad en la que han ocurrido los hechos, pandemia o estallido social, no han permitido a los protagonistas del sistema educativo, amoldarse a los cambios, trayendo como consecuencia que la mediación del aprendizaje cambió de ser cara a cara, interactiva entre docentes y estudiantes, entre estudiantes y sus pares, aquí y ahora(presencial) para intentar construir el aprendizaje a distancia que dependiendo como lo hayan planificado puede ser síncrono a través de chat ( WhatsApp, Skype, Messenger) o asíncrono mediante mensajería no instantánea, mensajes de texto, e-mail, entre otros, son cada vez más amigables. 
Cambian los insumos del sistema, las habilidades y destrezas de los estudiantes se ponen a prueba y se asume que son autorregulados, y la verdad la experiencia muestra que ni docentes ni estudiantes lo son, la autorregulación se da en tres fases referidas a:a) la experiencia o conocimiento previo del estudiante, que influyen y que se dan antes de los esfuerzos por aprender, preparar al estudiante para su proceso de aprendizaje. b) la ejecución o el control voluntario, que implica los procesos que se desarrollan cuando el participante decide autorregularse, toma conciencia y actúa en consecuencia, afectando la concentración y la ejecución. Y c) la autorreflexión que implica los procesos que tienen lugar después de aprender y que influyen sobre la reacción de lo aprendido hacia dicha experiencia. Este proceso permite sentar las bases para aprendizajes futuros (Zimmerman, 1998).

También se debe capacitar a los estudiantes para que aprendan a trabajar no solamente con TIC sino a través de las TIC, para ello se deben conocer sus competencias tecnológicas porque hay personas que asumen que por ser de cierta edad son expertos en el área y en realidad no es así, no todos son generación X o Milenial así tengan la edad para serlo, si no han estado en contacto con la tecnología no lo son.

El cambio de modalidad ha complicado las cosas para los actores del sistema por diferentes razones: a) el contenido que se ofrece no fue diseñado para ser administrado como curso a distancia b) no hay preparación previa ni docentes ni estudiantes para atender y estudiar cursos de educación a distancia) hay desapego por la falta de elementos sociales y experienciales que acompañan la experiencia presencial. Los docentes consideran que no tienen el equipamiento suficiente y adecuado para ejercer esta labor. A lo que Vargas, (2021), agrega que es primordial contacto cara a cara, la comunicación y la percepción de los estudiantes, para poder utilizar el sistema virtual.

Se cuenta con una variedad en los hallazgos en relación con la interacción del docente y el estudiante y las competencias a desarrollar.

El informante 1 (venezolana) señala que "los maestros han querido hacer sus clases a distancia sin tomar en cuenta el tiempo de atención ni la dificultad de estos, haciendo más difícil para los padres que los niños realicen sus tareas. Dado que la cantidad enorme de ellas molesta y recarga a los niños de trabajo durante largas horas". Informante 4 (venezolana en Zaragoza España) dice: "me gustan las clases virtuales de mi hija de prescolar, los saludan cada mañana y las asignaciones son tan variadas y dinámicas que la niña las hace con alegría. Menos mal que tenemos buen internet para que no se pierda ninguna interacción".

Algunos padres que no han entendido por diferentes razones, falta de motivación, tiempo o conocimiento tienden a dejar a la escuela toda la formación de sus niños y no participan ni en el desarrollo de las actividades de la casa y el momento actual exige que no solamente ayuden, sino que prácticamente cambien de rol y sean los maestros de sus hijos; y surge la pregunta, ¿tienen la formación para asumir ese rol? Otros aspectos negativos que se pueden señalar son padres sin preparación para acompañar en las tareas a sus hijos, el desconocimiento en el uso de los ambientes virtuales, la dificultad para usar el celular en el caso de varios niños, el costo de internet.

Además, en tiempo de diáspora de nuestros hermanos del país, hay muchas familiasde bajos recursos, o inmigrantes que necesitan escuelas abiertas porque tienen que trabajar y su empleador o rubro no le permiten trabajar desde casa, y llevar el sustento mientras que ahora deberán averiguar cómo participar en la educación en el hogar. Asimismo, la pérdida de ingresos conduce a que los segmentos vulnerables de la sociedad y las familias caigan por debajo de la línea de pobreza. Con el salario actual en Venezuela, por ejemplo, ninguna persona puede suplir sus necesidades básicas y poder honrar sus compromisos.

En cuanto a los docentes en ejercicio se requiere su actualización continua y permanente que disminuya la tensión y el desconocimiento y le permita enfrentar los cambios con apertura, aprovechando al máximo sus ventajas y potencialidades tanto en forma presencial o a distancia y así mejorar y optimizar su desempeño como mediador de procesos en sus funciones dentro del ámbito escolar. En los procesos de enseñanza y aprendizaje, creando redes de comunicación y nuevas necesidades, buscar giros y aplicaciones distintas.

Sobre la base del escenario descrito ya experiencia en la docencia de los autores, puede afirmarse que los docentes requieren apoyo prioritario, durante el período de confinamiento y en los procesos de reapertura de las escuelas, en al menos las siguientes áreas:

1. Formación continua y permanente, asesoría y recursos tecnológicos para trabajar en diferentes formatos de educación a distancia, incluida formación en competencias y metodologías para uso educativo de las TIC y otras plataformas de enseñanza y aprendizaje a distancia, y en criterios para la toma de decisiones curriculares contextualizadas y flexibles, evaluación y retroalimentación para el aprendizaje. 
2. Apoyo para mantener y profundizar los avances en la innovación metodológica y la implementación de formas alternativas de enseñanza, incorporando una apertura del currículo hacia lo lúdico, lo innovador, crítico y contextualizando la situación vivida, y en estrategias educativas para el aceleramiento y la recuperación de aprendizajes de los estudiantes.

3. Resguardo prioritario de la salud física y apoyo socioemocional, junto con el desarrollo de competencias para la enseñanza en materia de habilidades socio emocionales a los estudiantes y sus familias.

4. Fortalecimiento de las redes locales de profesorado mediante espacios de apoyo, aprendizaje y elaboración de propuestas colaborativas de abordaje del trabajo curricular, pedagógico y de apoyo socioemocional.En este sentido, el Desarrollo del Talento Humano del Personal Académico debe promover los procesos de actualización y perfeccionamiento del docente en el ejercicio de sus funciones, orientar hacia la profundización en su especialidad en lo referente a nuevos enfoques, tendencias, paradigmas y las TIC.

5. El reto para los docentes es autoformarse a través de cursos gratis vía internet o entrar en los cursos, talleres y medios que han ofrecido para ello en sus instituciones, de esa manera buscan soluciones creativas e innovadoras, actuando y algunos de ellos pues se han adaptado y rediseñado en función de los contenidos y diseños de los cursos para el aprendizaje en las distintas áreas de formación del docente se espera que: a) Tenga buenas habilidades comunicativas y de interacción, sepa mediar el aprendizaje en entornos digitales, b) use las redes sociales y las aplicaciones de los teléfonos celulares como herramientas educativas. c)desarrollo de medios entre ellos videos tutoriales, cuentos, historietas y guías instruccionales. d)desarrolle estrategias variadas e innovadoras para la mediación del aprendizaje. e) las tareas deben ser realistas o relevantes vinculadas con el mundo real y cotidiano que permita una comprensión del aprendizaje desde la experiencia particular de cada individuo, asignando un valor afectivo y emocional que es esencial en la motivación por descubrir y aprender. f) La interacción docente-padres de familia y el aprendiz debe ser cordial, natural, atractiva y práctica con el uso de objetos concretos de uso cotidiano, para realizar el acompañamiento u orientación efectiva g) además de proveer experiencias que los doten de herramientas que, utilizadas en situaciones reales o simuladas, garanticen su adecuada utilización para el desarrollo de las competencias específicas (Torres, Núñez, Caro \& Alvarado, 2018).

Al respecto Núñez, Gaviria-Serrano, Tobón, Guzmán-Calderón, \& Herrera (2019), señalan que"la práctica docente debe buscar en los estudiantes una mejor apropiación del saber a partir de estrategias novedosas y eficientes, no solo para el desarrollo de conocimientos teóricos, sino también de habilidades prácticas necesarias para el ejercicio profesional” (p. 2).

En cuanto a las estrategias y medios se deben tener en cuenta nuevas maneras de interacción docente-alumnos, alumnosalumnos, alumnos materiales electrónicos (CD, software, páginas Web) incorporar nuevas formas de encontrar, acceder y discriminar la información. Se deben diseñar y ejecutar las estrategias adaptando el discurso del profesor, y su expresión verbal y no verbal, para ello el modelaje es primordial. Además, las estrategias deben incentivar las experiencias colaborativas a través de grupos de discusión, chat o correos electrónicos.

Esto incluye acciones instruccionales relacionadas con el uso, selección, utilización y organización de la información de forma que el futuro docente vaya formándose como ciudadano de la sociedad de la información. Para un correcto uso de las TIC en la enseñanza, Monereo (2004), considera que el ciberespacio es una ventana que obliga a explicitar pensamientos y es el medio idóneo para desarrollar estrategias de aprendizaje, y el docente acompaña al educando como mediador para guiarlo en la toma de decisiones para ello se puede usar el modelado, la práctica guiada y luego la práctica autónoma haciendo que el educando la internalice y la aplique a cualquier situación. Esto implica hacer uso de los repositorios de las bibliotecas virtuales que de forma crítica y responsable para determinar qué usar y recomendar por parte del docente y en el caso del estudiante la búsqueda discriminada en función de la meta a realizar.

La evaluación de los aprendizajes es definida por Szczurek, (1989) como "el que tan bien" de los procesos de enseñanza y aprendizaje. El subsistema evaluación es el que determina el grado de eficacia y eficiencia de un sistema es el proceso mediante el cual se recopila información para emitir juicios de valor acerca de algo. Los entrevistados concuerdan con lo anterior y señalan además que la evaluación de los aprendizajes es justa, precisa, oportuna y está de acuerdo a las metas propuestas en el curso y es una evaluación que valora tanto el proceso como el producto para llevar al éxito. Al abordar la virtualización de la educación, no solamente es imprescindible es el uso de las TIC aplicadas a la misma, sino que cada uno de los actores educativos debe manejarlas con propiedad y tener los recursos info e infratecnológicos necesarios, además, deben participar activamente en situaciones educativas centradas en el alumno, o de intercambio de roles entre docentes y 
estudiantes, lo cual fomenta en el estudiante su autoaprendizaje, autorregulación, el desarrollo de su pensamiento crítico y creativo, mediante el trabajo en equipo cooperativo.

La UNESCO (2020) propone para la continuidad del aprendizaje:

1. Analizar el tipo de tecnología digital o no con la que se cuenta en la escuela y la comunidad y elegir la más pertinente.

2. Garantizar el carácter inclusivo de estudiantes con discapacidad o que proviene de familias de bajos ingresos en los programas de aprendizaje online

3. Proteger la privacidad y la seguridad de los datos de los estudiantes y docentes.

4. Crear vínculos entre las escuelas, los padres, los docentes y el estudiantado durante el confinamiento para darse apoyo socioemocional.

5. Planificar, ejecutar y valorar con frecuencia el desarrollo de los programas de aprendizaje online.

6. Proporcionar a docentes y estudiantes asistencia en cuanto al uso de las TIC, TAC y TEP para realizar las sesiones online, comunicarse y así poder garantizar las condiciones necesarias para que se realice la continuidad.

7. Combinar las teorías de aprendizaje, los enfoques adecuados y limitar la cantidad de aplicaciones y de plataformas. Se deben revisar las herramientas tecnológicas a los que el estudiante pueda tener acceso y evitar abrumarlos y a sus padres pidiéndoles que descarguen o prueben una gran cantidad de aplicaciones y plataformas.

8. Establecer las reglas de la educación online y dar seguimiento al proceso de aprendizaje, se debe definir entre docentes y las familias y el alumnado las reglas del aprendizaje online, es decir, cómo se resolverán dudas, cómo se realizarán los ejercicios y se evaluarán, etc. Y las herramientas de comunicación para transmitir sus comentarios 0 preguntas al profesorado. No es lo mismo trabajar con entornos virtuales para adultos que para niños, niñas y adolescentes.

9. Definir el tiempo de duración de las clases online en función del alumnado

10. Crear comunidades virtuales de docentes, familias y directores de centros educativos para facilitar los intercambios de experiencias, entre el profesorado y favorecer los vínculos sociales.

En fin, para usar un entorno virtual es primordial apropiarse de esos espacios con las condiciones de formación de los actores del sistema: docente, estudiantes y los recursos necesarios para el intercambio de información, la generación de conocimientos y nuevas experiencias.

En la situación política, económica y social de la Venezuela de hoy; hay más preguntas que respuestas a veces nos preguntamos ¿cuál es el venezolano que se busca?, la respuesta está en nuestra cultura, valores principios y definitivamente en nuestro marco legal y es allí donde está nuestro norte y definitivamente la base para la formación del ciudadano que necesitamos y que la escuela debe forjar con competencias básicas referidas a aquellas actitudes, destrezas y conocimientos más elementales que permiten ejercer al ser humano alguna actividad durante la cotidianidad o el día a día y competencias transversales, entendidas en todas sus dimensiones y pueden ser empleadas en cualquier ámbito laboral. En otras palabras, son habilidades superiores que posee un individuo y su puesta en práctica le permite desarrollar alguna actividad en un entorno organizativo (Cinterfor; 2004 p. 12).

\section{CONCLUSIONES}

Existe una gran variedad de efectos de la pandemia en el ámbito educativo, hay países que se sobreponen y han tratado de subsanar las fallas, no así Venezuela, cada vez más se muestra la improvisación y la poca posibilidad que tienen los docentes por el contexto socio económico y social del país. Es importante avanzar hacia el desarrollo en sus estudios de lo que podemos extraer que la importancia de la educación y formación gratuita de docentes y administradores, además de resaltar la importancia de la educación ahora y en el futuro, el papel de las féminas en la difusión del conocimiento en relación con el covid19.

La tarea de la planificación de los procesos de enseñanza y aprendizaje, los problemas y estadísticas correspondientes y las soluciones implementadas por las diferentes regiones en estos momentos de pandemia. Durante el año 2020 hubo muchos cambios en diferentes niveles ambiental, económico, social y por ende el educativo con cambios relevantes, bruscos y en muchos casos sin planificación ni valoración efectiva de las consecuencias que han ocasionado.

Los maestros con poca o ninguna formación en educación a distancia que a vapor han desarrollado, planificado y ejecutado planificaciones y evaluaciones sin medir las consecuencias en la formación integral de los niños y jóvenes. También sin 
tomar en cuenta que el desarrollo de todas las competencias puede hacerse a distancia caso de laboratorios. Se ha forzado el trabajo académico y en muchos casos el proceso de aprendizaje.

Se requiere el análisis y evaluación de las fortalezas y amenazas que representan los problemas y alcances que la educación distancia ha evidenciado durante la crisis actual, para superar las deficiencias pedagógicas y la agudización de la exclusión y la desigualdad social y de género, en los modelos de enseñanza virtual y de sistemas híbridos (presenciales y a distancia) para la docencia. Esto sin añadir el vacío o disminución en el ámbito de la investigación, en las ciencias y las humanidades.

\section{Conflicto de intereses}

El autor declara que no existe conflicto de intereses

\section{REFERENCIAS}

Amitabh, U. (Agosto 31, 2020). Cómo la tecnología transformará el aprendizaje en la era COVID19. Agenda Global. https://www.weforum.org/agenda/2020/08/howedtech-will-transformlearning-in-the-covid-19-era/

Centro Interamericano para el Desarrollo del Conocimiento en la Formación Profesional Cinterfor (2004, abril) 0 Preguntas sobre competencia laboral. Documentos sobre avances sobre competencias laborales en la región. (2da Edición). Uruguay: Autor. [Documento en línea], Disponible: http://www.oitcinterfor.org/publicaci\%C3\%B3n/40-preguntas-sobre-competencia-laboral

Ferrada-Bustamante, V., González-Oro, N., Ibarra-Caroca, M., Ried-Donaire, A., Vergara-Correa, D., \& Castillo-Retamal, F. (2021). Formación docente en TIC y su evidencia en tiempos de COVID-19. Revista Saberes Educativos, (6), 144-168. https://doi.org/10.5354/2452-5014.2021.60715

García, M. (24 de Julio de 2020). La docencia desde el hogar. Una alternativa necesaria en tiempos del Covid 19. Polo del Conocimiento, 5(4), 304-324. https://dialnet.unirioja.es/servlet/articulo?codigo=7398376

Guzmán, B, (2020). Educación y las TIC en tiempos difíciles. [conferencia]Postdoctorado: el currículum y la transcomplejidad ante los desafíos de la contemporaneidad. Caracas, Venezuela

Guzmán, B., \& Castro, S. (2020). Los medios instruccionales, su desarrollo e importancia en la educación del siglo XXI. Delectus, 3(1), 1-16. https://doi.org/10.36996/delectus.v3i1.35

Guzmán, B. (2013). Evaluación del Curso Tecnología de Información y Comunicación y Educación Ambiental a Través de sus Productos. (Trabajo de Ascenso para optar a la categoría de profesor Titular). Universidad Pedagógica Experimental Libertador, Instituto Pedagógico de Caracas.

Marinoni, G., Van'tLand, H., \& Jensen, T. (2020). The Impact of COVID-19 on Higher Education Around the World IAU. http://www.educacionyfp.gob.es/dam/jcr:08a58c10-1617-4103-8518-2cb823f38fa9/impact-of-covid-19.pdf.

Monereo, C., (2004) El Aprendizaje Estratégico En La Sociedad Del Conocimiento. Conferencia presentada en el evento I encuentro sobre aprendizaje estratégico. UPEL-IPC, Caracas Venezuela 26- 30 de Julio de 2004.

Núñez, C; Gaviria-Serrano, j, Tobón, Guzmán-Calderón, C, Herrera, S R. 2019La práctica docente mediada por TIC: una construcción de significados Revista ESPACIOS. Vol. 40 ( $\mathrm{N}^{\circ}$ 5) https://revistaespacios.com/a19v40n05/19400504.html\#

Ojeda N., \& Palacios Y., (2021) Relación intrínseca del liderazgo, tecnologías, COVID y educación, un espacio de reflexión para situaciones pandémicas. Revista de Investigación, 45(102), 96-121.https://es.calameo.com/books/00624679990cc578b80bb

Ordorika, I. (2020). Pandemia y educación superior. Revista De La Educación Superior, 49(194), 1-8. http://resu.anuies.mx/ojs/index.php/resu/article/view/1120

Picón, G., González de Caballero, G., \& Paredes Sánchez J. (2020). Desempeño y formación docente en competencias digitales en $\begin{array}{lllll}\text { clases no presenciales durante la pandemia } & \text { COVID-19. }\end{array}$ https://preprints.scielo.org/index.php/scielo/preprint/download/778/1075/1115

Piñero González F., (2021) El viraje educativo, de la presencialidad a la Educación a Distancia en tiempos de pandemia (COVID-19). Revista de Investigación, 45(102), 286-30.https://es.calameo.com/books/00624679990cc578b80bb

Puleo, A. (2020). Reflexiones Ecofeministas Ante La Pandemia de COVID-19. The Conversation. https://theconversation.com/reflexiones-ecofeministas-ante-la-pandemia-de-covid-19-135159

Reynosa Navarro, E., Guerra Ayala, M. J., Casimiro Urcos, W. H., Vélez-Jiménez, D., Casimiro-Urcos, N. C., Salazar Montoya., E. O., Casimiro-Urcos, J. F., \& Callejas Torres, J. C. (2021). Relevance of the mass media in Prevention, Education and Contextual Management of COVID-19. World Journal on Educational Technology: Current Issues, 13(1), 129-146. https://doi.org/10.18844/wjet.v13i1.5423 
Rogero-García, J., (2020). La ficción de educar a distancia. Revista de Sociología de la Educación-RASE, 13(2). 174-182. https://doi.org/10.7203/RASE.13.2.17126

Rujas, J., \& Feito R., (2021)La educación en tiempos de pandemia: una situación excepcional y cambiante. Revista de Sociología de la Educación-RASE, 14(1), 4-13. https://doi.org/10.7203/RASE.14.1.20273

Szczurek, M. (1989). La estrategia instruccional. Investigación y Postgrado, 4. (2). 7-26.

Torres, R., Núñez, C., Caro, f. \& Alvarado, P. (2018, en prensa). La dinámica del conocimiento. Una integración entre las prácticas de aula y el desempeño académico. En Nuñez, C., Barzotto, V., Tobón, S. (Eds.). Escuchando las voces en aulas escolares. Experiencias significativas y transformación de las prácticas docentes. Medellín: Sello Editorial Universidad de Medellín http://hdl.handle.net/11407/6206

UNESCO IESALC, (2020). COVID-19 y educación superior: De los efectos inmediatos al día después. http://www.iesalc.unesco.org/wp-content/uploads/2020/05/COVID-19-ES- 130520.pdf

UNESCO. (Abril 1, 2020). Propuestas de la UNESCO Para garantizar la educación online durante la pandemia. Redacción de Educaweb. https://www.educaweb.com/noticia/2020/04/01/propuestas-unesco-garantizar-educacion-online-pandemia-19132/

Vargas, F. (2021). La vivencia de la educación a distancia y estrategias de enseñanza y aprendizaje en tiempos de pandemia, desde la voz de sus protagonistas. Aula virtual, 2(4), 114-126. http://aulavirtual.web.ve/revista/ojs/index.php/aulavirtual/article/view/69

Villén, C. (2020). El profesorado y las tecnologías en tiempos de confinamiento por la pandemia Covid-19. Creencias sobre actitudes, formación, competencia digital e importancia de las TIC en educación. [Tesis de Maestría, Universidad de Salamanca]. https://gredos.usal.es/bitstream/handle/10366/143691/TFM_Vill\%C3\%A9nS\%C3\%A1nchezC_Profesoradoytecnolog\%C3\%A Das.pdf?sequence $=1$ \&isAllowed $=\mathrm{y}$

Zimmerman, B. J. (1998). Developing self-fulfilling cycles of academic regulation: An analysis of exemplary instructional model. En D. H. Shunck y B. J. Zimmerman (Eds.), Self-Regulated learning: From teaching to self-reflective practice (pp. 1-19). Nueva York, NY: GuilfordPress. 